\title{
On a somewhat forgotten condition of Hasegawa and on Blackwell's example
}

\author{
ADAM BoBRowsKI
}

\begin{abstract}
We show, by presenting two examples, that a somewhat forgotten condition of Hasegawa (Proc Jpn Acad 40:262-266, 1964) is useful in proving convergence of operator semigroups, and may be more handy than the standard range condition. Also, we present the semigroup related to Blackwell's example (Ann Math Statist 29:313-316, 1958) as an infinite product of commuting Markov semigroups. Intriguingly, it is hard to find a manageable description of the generator of this semigroup. As a result, it is much easier to prove the existence of the infinite product involved by direct argument than it is to do this using the Trotter-KatoSova-Kurtz-Hasegawa theory.
\end{abstract}

Mathematics Subject Classification. 47D06, 47D09.

Keywords. Infinite product of semigroups of operators, Blackwell's example, Range condition.

1. Introduction. Let $\left(\mathrm{e}^{t A_{n}}\right)_{t>0}, n \geq 1$ be a sequence of equibounded semigroups of linear operators in a Banach space $\mathbb{X}$, generated by $A_{n}$, i.e. suppose there exists an $M>0$ such that

$$
\left\|\mathrm{e}^{t A_{n}}\right\|_{\mathcal{L}(\mathbb{X})} \leq M, \quad n \geq 1, t \geq 0
$$

The classical Trotter-Kato-Neveu theorem ([1] p. 149, [4] p. 342, [7] p. 212, or [11] p. 44) says that these semigroups converge in the strong topology iff

(a) for all $\lambda>0$, the resolvents $\left(\lambda-A_{n}\right)^{-1}=\int_{0}^{\infty} \mathrm{e}^{-\lambda t} \mathrm{e}^{t A_{n}} \mathrm{~d} t$ converge in the strong topology, and

(b) the range of the limit pseudo-resolvent $R_{\lambda}:=\lim _{n \rightarrow \infty}\left(\lambda-A_{n}\right)^{-1}, \lambda>0$ (which does not depend on $\lambda$ ) is dense in $\mathbb{X}$. 
More specifically, in this case $R_{\lambda}, \lambda>0$ is the resolvent of a densely defined closed operator $A$, which turns out to be the generator of the limit semigroup:

$$
\lim _{n \rightarrow \infty} \mathrm{e}^{t A_{n}}=\mathrm{e}^{t A}
$$

strongly and almost uniformly in $t \geq 0$.

An equivalent formulation of this result, due to M. Sova and T.G. Kurtz ([8, $16,17,21]$ p. 28 , [4] p. 345), uses the notion of the extended limit of operators $A_{n}, n \geq 1$ : the extended limit is a (possibly multivalued) operator $A_{\text {ex }}$ defined by

$$
(x, y) \in A_{\mathrm{e} x} \quad \text { iff } \quad \exists_{x_{n} \in D\left(A_{n}\right)} \lim _{n \rightarrow \infty}\left(x_{n}, A_{n} x\right)=(x, y),
$$

where $(x, y) \in \mathbb{X} \times \mathbb{X}$ and the Cartesian product space is equipped with one of usual, equivalent norms. It transpires that condition (a) in the Trotter-KatoNeveu theorem holds iff (a)' for some (and then for all) $\lambda>0$, the range of $\lambda-A_{\mathrm{e} x}$ equals $\mathbb{X}$, and then (b) holds iff $D\left(A_{\mathrm{e} x}\right)$ is dense in $\mathbb{X}$. More specifically, $A_{\mathrm{ex}}$ turns out to be single-valued and is the generator of the limit semigroup.

In particular, the density of $D\left(A_{\mathrm{e} x}\right)$ in $\mathbb{X}$ does not guarantee convergence of semigroups; it must be accompanied by the range condition (a)', which often is the hardest to establish. In fact, proving the range condition is as difficult as proving that a certain operator is the generator of a semigroup.

Fortunately, sometimes checking the range condition may be avoided if one is able to check the alternative condition of Hasegawa [12]. The latter is

$$
\lim _{n, m \rightarrow \infty}\left\|\mathrm{e}^{s A_{n}} \mathrm{e}^{t A_{m}} x-\mathrm{e}^{t A_{m}} \mathrm{e}^{s A_{n}} x\right\|=0, \quad x \in \mathbb{X}
$$

almost uniformly in $s, t \geq 0$, and its weaker version in terms of resolvents is

$$
\lim _{n, m \rightarrow \infty}\left\|\left(\lambda-A_{n}\right)^{-1}\left(\lambda-A_{m}\right)^{-1} x-\left(\lambda-A_{m}\right)^{-1}\left(\lambda-A_{n}\right)^{-1} x\right\|=0,
$$

for $\lambda>0, x \in \mathbb{X}$. More specifically, we have the following theorem.

Theorem 1.1 (Hasegawa [12]). The semigroups $\left(\mathrm{e}^{t A_{n}}\right)_{t \geq 0}$ converge on $\mathbb{X}$ iff the set $D\left(A_{\mathrm{e} x}\right)$ is dense in $\mathbb{X}$ and condition (1.2) holds.

The aim of this paper is to show that this, somewhat forgotten, condition is sometimes satisfied automatically, and then the analysis based on Hasegawa's theorem is much easier than that based on the classical Trotter-Kato-Neveu theorem or its Sova-Kurtz version. To this end, we present two examples: one involves the question of the existence of infinite products of semigroups [2], the other concerns the existence of a boundary value of a holomorphic semigroup [1] p. 174.

The third example, discussed in the second part, is of slightly different nature. Namely, we present a semigroup-theoretical approach to Blackwell's famous example [3] of a Markov chain with denumerably many states all of which are instantaneous. The point is that although this example fits well into the scheme of infinite products of semigroups, and despite the fact that all generators involved are bounded, it is quite difficult to check that $D\left(A_{\mathrm{e} x}\right)$ is dense, and the whole machinery of the theory of convergence of semigroups fails. (This is the reflection of the fact that it is quite difficult to find explicitly 
a single non-zero vector in the domain of the generator of the limit semigroup.) On the other hand, convergence of the semigroups involved may be established directly — what does not happen too often either.

It should be perhaps noted here that examples of semigroups given by explicit formulae, where it is hard to characterize the generator in a full way, are known and include for instance the Cauchy semigroup (called also a Poisson semigroup) in the space of continuous functions [22, Chapter 10] (but not in the $L^{p}$ spaces, see [19, p. 99]). However, in these examples one is at least able to find a core of the generator; moreover, the form of the generator on the core is known (see e.g. Hunt's theorem on generators of convolution semigroups [13] p. 262, [14]). In Blackwell's example it is difficult to find a single non-zero member of the domain, not to mention the form of the generator.

2. Infinite product of commuting semigroups. Let us consider the question of existence of an infinite product of commuting contraction semigroups. This question was first discussed in Arendt et al. [2], a motivating example being the heat semigroup in infinite dimensions introduced by Cannarsa and Da Prato [5]. Let $B_{n}, n \geq 1$ be generators of commuting contraction semigroups:

$$
\mathrm{e}^{t B_{n}} \mathrm{e}^{s B_{k}}=\mathrm{e}^{s B_{k}} \mathrm{e}^{t B_{n}}, \quad s, t \geq 0, k, n \geq 1 .
$$

Then $\left(T_{n}(t)\right)_{t \geq 0}$ where $T_{n}(t)=\prod_{k=1}^{n} \mathrm{e}^{t B_{k}}$ is a strongly continuous contraction semigroup with the generator $A_{n}=\overline{\sum_{k=1}^{n} B_{k}}$, the closure of $\sum_{k=1}^{n} B_{k}$ defined on $\bigcap_{k=1}^{n} D\left(B_{k}\right)$ (see e.g., [20, A-1.3.8]). We say that the infinite product $T(t)=$ $\prod_{k=1}^{\infty} \mathrm{e}^{t B_{k}}$ exists iff the semigroups $\left(T_{n}(t)\right)_{t \geq 0}$ converge.

The following theorem from [2] was proved there in a different way, the authors being apparently unaware of Hasegawa's result.

Theorem 2.1. Define an operator $A$ by $A x=\sum_{k=1}^{\infty} B_{k} x$ with domain

$$
D(A)=\left\{x \in \bigcap_{k=1}^{\infty} D\left(B_{k}\right) ; \sum_{k=1}^{\infty}\left\|B_{k} x\right\|<\infty\right\}
$$

and suppose $D(A)$ is dense in $\mathbb{X}$. Then, the infinite product of $\left(\mathrm{e}^{t B_{n}}\right)_{t \geq 0}$ exists and its generator is the closure of $A$.

Proof. Clearly, $A$ is a restriction of $A_{\mathrm{e} x}$ and the assumption implies that $D\left(A_{\mathrm{e} x}\right)$ is dense in $\mathbb{X}$. Since $\left(\mathrm{e}^{t A_{n}}\right)_{t>0}$ commute, Hasegawa's condition is satisfied and the infinite product exists by Theorem 1.1. Also, the generator of the product, equaling $A_{\mathrm{e} x}$, is an extension of $A$. Hence, we are left with proving that $D(A)$ is a core for $A_{\mathrm{e} x}$. To this end, by the well-known condition for a subset of a generator to be a core (see e.g., [4, p. 261], [7, p. 53], or [8, Proposition $3.3]$ ), it suffices to show that $D(A)$ is left invariant by $T(t)=\prod_{k=1}^{\infty} \mathrm{e}^{t B_{k}}$.

Fix $k \in \mathbb{N}$ and $x \in D\left(B_{k}\right)$. For any $n \in \mathbb{N}$ and $t \geq 0, T_{n}(t) x$ belongs to $D\left(B_{k}\right)$ and $B_{k} T_{n}(t) x=T_{n}(t) B_{k} x$ since $\left(\mathrm{e}^{t B_{k}}\right)_{t \geq 0}$ commutes with $\left(T_{n}(t)\right)_{t \geq 0}$. Closedness of $B_{k}$ implies now $T(t) x \in D\left(B_{k}\right)$ and $B_{k} T(t) x=T(t) B_{k} x$. Thus, for $x \in D(A), T(t) x$ belongs to $\bigcap_{k=1}^{\infty} D\left(B_{k}\right)$ and $\sum_{k=1}^{\infty}\left\|B_{k} T(t) x\right\| \leq$ $\sum_{k=1}^{\infty}\left\|B_{k} x\right\|<\infty$, proving that $T(t) x \in D(A)$. 
3. Boundary value of a holomorphic semigroup. As a second example, we establish existence of the boundary value of a holomorphic semigroup. To recall, a closed operator $A$ in a complex Banach space is said to be sectorial of angle $\delta_{0} \in\left(0, \frac{\pi}{2}\right]$ if the sector

$$
\Sigma_{\frac{\pi}{2}+\delta_{0}}=\left\{\lambda \in \mathbb{C} \backslash\{0\} ;|\arg \lambda|<\frac{\pi}{2}+\delta_{0}\right\}
$$

is contained in the resolvent set $\rho(A)$ of $A$, and for all $\delta \in\left(0, \delta_{0}\right)$ there exists $M_{\delta}>0$ such that

$$
\left\|(\lambda-A)^{-1}\right\| \leq \frac{M_{\delta}}{|\lambda|}, \quad \lambda \in \Sigma_{\frac{\pi}{2}+\delta} .
$$

If $A$ is additionally densely defined (see [1] pp. 157-158, [7] p. 96, [15] p. 488 or [24] p. 257), it generates a strongly continuous semigroup $\left(\mathrm{e}^{t A}\right)_{t>0}$ which may be extended to the complex $t$ in $\Sigma_{\delta_{0}}$. For $t \in \Sigma_{\delta}$, where $\delta<\delta_{0}$, the semigroup may be expressed as the Dunford integral:

$$
\mathrm{e}^{t A}=\frac{1}{2 \pi i} \int_{\gamma} \mathrm{e}^{t \lambda}(\lambda-A)^{-1} \mathrm{~d} \lambda
$$

where $\gamma$ is a piecewise smooth curve running, within the closure of $\Sigma_{\frac{\pi}{2}+\delta}$ (excluding $\lambda=0$ ), i.e., within

$$
\left\{\lambda \in \mathbb{C} \backslash\{0\} ;|\arg \lambda| \leq \frac{\pi}{2}+\delta\right\}
$$

from infinity with $\arg \lambda=-\left(\frac{\pi}{2}+\delta\right)$ to infinity with $\arg \lambda=\frac{\pi}{2}+\delta$. By (3.1), the integral here is absolutely convergent and by Cauchy's theorem it does not depend on the choice of the curve. Moreover, $\lim _{t \rightarrow 0, t \in \Sigma_{\delta}} \mathrm{e}^{t A} x=x$ for all $x \in \mathbb{X}$ and $\delta \in\left(0, \delta_{0}\right)$, and the semigroup is bounded in each sector $\Sigma_{\frac{\pi}{2}+\delta}, \delta \in\left(0, \delta_{0}\right)$. Hence, $A$ is said to generate a bounded holomorphic semigroup.

Let $A$ be sectorial of angle $\delta_{0}$. Then for $z \in \mathbb{C}$ with $|z|=1$ and $|\arg z|<\delta_{0}$, $z A$ generates a strongly continuous semigroup $\left(\mathrm{e}^{t z A}\right)_{t \geq 0}$. This, however, does not need to be so if $\arg z=\delta_{0}$. The question of existence of the semigroup generated by $z_{0} A$ where $z_{0}=\mathrm{e}^{i \delta_{0}}$ is known as that of existence of the boundary value of a holomorphic semigroup. It turns out that the boundary value semigroup exists iff

$$
K:=\sup _{t \in \Sigma_{\delta_{0}}, \Im t \geq 0,|t| \leq 1}\left\|\mathrm{e}^{t A}\right\|
$$

is finite. A simple proof of necessity of this condition may be found in $[1, \mathrm{p}$. 174], and we will restrict ourselves to proving the sufficiency.

Let $z_{n}$ satisfy $\left|z_{n}\right|=1,\left|\arg z_{n}\right|<\delta_{0}$, and $\lim _{n \rightarrow \infty} z_{n}=z_{0}$. By assumption, $\left\|\mathrm{e}^{t z_{n} A}\right\| \leq K$ for $t \in[0,1]$ and $n \geq 1$. This implies that there exist $M \geq 1$ and $\omega \in \mathbb{R}$ such that $\left\|\mathrm{e}^{t z_{n} A}\right\| \leq M \mathrm{e}^{\omega t}, t \geq 0, n \geq 1$, and the semigroups generated by $A_{n}=z_{n} A-\omega I$ are equibounded. The semigroups generated by $z_{n} A$, being 'cut out of' the single semigroup generated by $A$, commute. Hence, the operators $A_{n}$ satisfy the Hasegawa condition. Also $\lim _{n \rightarrow \infty} A_{n} x=$ $z_{0} A x-\omega x$ for $x \in D(A)$, and $D(A)$ is dense by assumption. Therefore, the strong limit $\lim _{n \rightarrow \infty} \mathrm{e}^{t A_{n}}$ exists by Theorem 1.1, the generator of the limit 
semigroup is the extended limit $A_{\mathrm{e} x}$ of $A_{n}$, and $z_{0} A-\omega I \subset A_{\mathrm{e} x}$. Since all $z_{n} \neq 0$, closedness of $A$ implies $A_{\mathrm{e} x} \subset z_{0} A-\omega I$. Thus $z_{0} A=\omega I+A_{\mathrm{e} x}$ generates a semigroup as well.

4. Blackwell's example. We turn to the discussion of Blackwell's example of a honest Markov chain with countably many states all of which are instantaneous. (See the original paper [3] or e.g., [10, p. 297] or [18, p. 65]. Predecessors of this example may be found in $[6,9]$.) The example involves the infinite product of commuting semigroups of Section. 2; intriguingly, it is easier to prove the existence of this product directly, by finding an explicit formula, than it is to do it by using Theorem 2.1. In fact, in notations of Theorem 2.1, it is quite difficult to find a single, non-zero vector $x \in D(A)$. It's even worse: it is difficult to find a non-zero $x$ for which $\sum_{n=1}^{\infty} B_{n} x$ converges, despite the fact that all $B_{n}$ 's are bounded.

To begin the presentation of the example, let $S$ be the set of functions $s: \mathbb{N} \rightarrow\{0,1\}$ admitting value 1 finitely many times. Since $S$ is countable, functions $x: S \rightarrow \mathbb{R}$ will be called sequences. By $l^{1}$ we denote the space of absolutely summable sequences on $S: x$ is a member of $l^{1}$ if $\|x\|:=\sum_{s \in S}|x(s)|<\infty$. We note that the members $\delta_{s}, s \in S$ of $l^{1}$ defined by

$$
\delta_{s}\left(s^{\prime}\right)= \begin{cases}1, & s^{\prime}=s \\ 0, & s^{\prime} \neq s\end{cases}
$$

form a Schauder basis for this space: in particular, for each $x \in l^{1}$,

$$
x=\sum_{s \in S} x(s) \delta_{s} .
$$

Given positive numbers $\alpha_{n}, \beta_{n}, n \geq 1$ we construct a sequence of Markov semigroups in $l^{1}$. To this end, we need some notation. For $n \geq 1$ let $F_{n}$ be the map $F_{n}: S \rightarrow S$ changing the $n$-th coordinate of an $s$ from 0 to 1 and vice versa. Also, for a finite set $E=\left\{i_{1}, \ldots, i_{k}\right\} \subset \mathbb{N}$, let

$$
F_{E}:=F_{i_{1}} \circ \cdots \circ F_{i_{k}}
$$

be the map that changes an $s \in S$ at its coordinates $i_{1}, \ldots, i_{k}$. Finally, let $G_{n}: S \rightarrow\{0,1\}$ assign to an $s$ its $n$-th coordinate.

For $n \geq 1$, let $B_{n}$ be the bounded linear operator determined by its values on the Schauder basis by

$$
B_{n} \delta_{s}= \begin{cases}-\beta_{n} \delta_{s}+\beta_{n} \delta_{F_{n}(s)}, & \text { if } G_{n}(s)=0, \\ -\alpha_{n} \delta_{s}+\alpha_{n} \delta_{F_{n}(s)}, & \text { if } G_{n}(s)=1 .\end{cases}
$$

$B_{n}$ is the generator of a Markov chain on $S$ in which the $n$-th coordinate of an $s \in S$ jumps between 0 and 1 , the intensity of the 'forward' jump being $\beta_{n}$ and that of the 'backward' jump being $\alpha_{n}$. In other words, $B_{n}$ is the generator of the Markov semigroup in $l^{1}$ determined by

$$
\mathrm{e}^{t B_{n}} \delta_{s}= \begin{cases}p_{n}(t) \delta_{s}+\left(1-p_{n}(t)\right) \delta_{F_{n}(s)}, & \text { if } G_{n}(s)=0 \\ q_{n}(t) \delta_{s}+\left(1-q_{n}(t)\right) \delta_{F_{n}(s)}, & \text { if } G_{n}(s)=1\end{cases}
$$


where

$$
\begin{aligned}
& p_{n}(t)=\frac{\alpha_{n}}{\alpha_{n}+\beta_{n}}+\frac{\beta_{n}}{\alpha_{n}+\beta_{n}} \mathrm{e}^{-\left(\alpha_{n}+\beta_{n}\right) t}, \\
& q_{n}(t)=\frac{\beta_{n}}{\alpha_{n}+\beta_{n}}+\frac{\alpha_{n}}{\alpha_{n}+\beta_{n}} \mathrm{e}^{-\left(\alpha_{n}+\beta_{n}\right) t} .
\end{aligned}
$$

(The term 'Markov' refers to the fact that the semigroup preserves the set of positive vectors of norm one.) It is clear that $B_{n}$ 's commute, so that we are in the setup of the first example of Section. 2, i.e., in the setup of [2]. Moreover, since the $B_{n}$ 's are bounded, the generator of the strongly continuous semigroup $T_{n}(t)=\prod_{k=1}^{n} \mathrm{e}^{t B_{k}}$ is $A_{n}=\sum_{k=1}^{n} B_{k}$. This semigroup describes $n$ combined independent Markov chains, each changing one of the first $n$ coordinates of $s$ as described above, and is determined by

$$
T_{n}(t) \delta_{s}=\sum_{E \subset\{1, \ldots, n\}} p_{E, n}(t, s) \delta_{F_{E}(s)}
$$

with summation over $2^{n}$ subsets $E$ of $\{1, \ldots, n\}$ and

$$
p_{E, n}(t, s)=\prod_{i=1}^{n} r_{i}(t, s, E),
$$

where

$$
r_{i}(t, s, E)= \begin{cases}p_{i}(t), & \text { if } G_{i}(s)=0 \text { and } i \notin E, \\ 1-p_{i}(t), & \text { if } G_{i}(s)=0 \text { and } i \in E, \\ q_{i}(t), & \text { if } G_{i}(s)=1 \text { and } i \notin E, \\ 1-q_{i}(t), & \text { if } G_{i}(s)=1 \text { and } i \in E .\end{cases}
$$

This formula simply says that, if the initial state of the Markov chain related to $A_{n}$ is given, its state at time $t \geq 0$ may be characterized by listing the coordinates which are different from the original ones - this is the role of the set $E$. Since each coordinate evolves independently from the other ones, the probability of such a change is the product of probabilities of change or no change on the first, second, third, and remaining coordinates.

Assume now that

$$
\sum_{n=1}^{\infty} \frac{\beta_{n}}{\alpha_{n}+\beta_{n}}<\infty,
$$

and fix $s$ and a finite subset $E$ of $\mathbb{N}$. Then, for $n$ large enough, $E \subset\{1, \ldots, n\}$. Also, since $s$ has finitely many coordinates different from 0 , for sufficiently large $n$ and $i>n, r_{i}(t, s, E)$ describes the probability of no change from initial 0 in the $i$-th coordinate of $s$ (after perhaps many changes back and forth in the meantime), i.e., $r_{i}(t, s, E)=p_{i}(t)$. Recalling that the product $\prod_{n=1}^{\infty} a_{n}$, where $a_{n} \in(0,1]$, converges if so does the series $\sum_{n=1}^{\infty}\left(1-a_{n}\right)$, we see that the limit

$$
p_{E}(t, s):=\lim _{n \rightarrow \infty} p_{E, n}(t, s)
$$


exists because the finite number of terms in the product does not influence convergence, while

$$
\sum_{n=1}^{\infty}\left(1-p_{n}(t)\right)=\sum_{n=1}^{\infty} \frac{\beta_{n}}{\alpha_{n}+\beta_{n}}\left(1-\mathrm{e}^{-\left(\alpha_{n}+\beta_{n}\right) t}\right) \leq \sum_{n=1}^{\infty} \frac{\beta_{n}}{\alpha_{n}+\beta_{n}}<\infty,
$$

by assumption.

Lemma 4.1. As $n \rightarrow \infty$, the right-hand side of (4.2) converges to

$$
T(t) \delta_{s}:=\sum_{E} p_{E}(t, s) \delta_{F_{E}(s)},
$$

where the sum is over all finite subsets $E$ of $\mathbb{N}$.

Proof. Clearly, $\sum_{E \subset\{1, \ldots, n\}} p_{E, n}(t, s)=1$ for $s \in S, t \geq 0$ and $n \geq 1$, and the summands are non-negative. Therefore, by (4.5) and Scheffé's lemma (see e.g., $[23$, p. 55] or $[4$, p. 199]), all we need to show is that

$$
\sum_{E} p_{E}(t, s)=1, \quad \text { for } \quad t \geq 0, s \in S .
$$

The proof of this relation will be more transparent if we assume $s=(0,0, \ldots)$; this we do without loss of generality. Also, for simplicity of notation, we suppress dependence on $t$ and $s$, and write $p_{n}$ and $p_{E}$ instead of $p_{n}(t)$ and $p_{E}(t, s)$, respectively. Thus

$$
p_{E}=\prod_{n \in E}\left(1-p_{n}\right) \prod_{n \notin E} p_{n}
$$

For a (finite) $E \subset \mathbb{N}$, let

$$
E_{1}= \begin{cases}E \backslash\{1\}, & \text { if } 1 \in E, \\ E \cup\{1\}, & \text { if } 1 \notin E .\end{cases}
$$

Then

$$
p_{E}+p_{E_{1}}=\prod_{n \in E, n \neq 1}\left(1-p_{n}\right) \prod_{n \notin E, n \neq 1} p_{n} .
$$

Therefore, letting $L=\sum_{\text {finite } E \subset\{1,2, \ldots\}} p_{E}$, we have

$$
L=\sum_{\text {finite }} \prod_{E \subset\{2,3, \ldots\}}\left(1-p_{n}\right) \prod_{n \in E} p_{n} .
$$

Repeating this argument $k$ times, we see that

$$
L=\sum_{E} \prod_{n \in E}\left(1-p_{n}\right) \prod_{n \in(\mathbb{N} \backslash\{1, \ldots, k\}) \backslash E} p_{n},
$$

where $E \subset\{k+1, k+2, \ldots\}$ are finite sets. Since the sum includes the component corresponding to $E=\emptyset, L \geq \prod_{n=k+1}^{\infty} p_{n}$. On the other hand, the last product may be chosen as close to 1 as we wish by taking $k$ large enough. This completes the proof. 
Theorem 4.2. The semigroups $\left(T_{n}(t)\right)_{t \geq 0}$ converge to the semigroup $(T(t))_{t \geq 0}$;

$$
T(t) x=\sum_{s \in S} x(s) T(t) \delta_{s}
$$

where $T(t) \delta_{s}$ is defined in Lemma 4.1. The limit semigroup is strongly continuous and is composed of Markov operators.

Proof. The statement concerning convergence is clear by Lemma 4.1. Also, $(T(t))_{t \geq 0}$ is a semigroup, being a limit of semigroups, and its Markovian nature is a direct consequence of (4.6). Hence, we are left with showing that $(T(t))_{t \geq 0}$ is strongly continuous, and for this it suffices to show that $\lim _{t \rightarrow 0} T(t) \delta_{s}=$ $\delta_{s}, s \in S$. Let $s \in S$, and let $k$ be so large that all coordinates of $s$ with indexes larger than $k$ are zeros. Then

$$
p_{\emptyset}(t, s)=\prod_{i=1}^{n} r_{i}(t, s, \emptyset) \prod_{i=n+1}^{\infty} p_{i}(t) \geq \prod_{i=1}^{n} r_{i}(t, s, \emptyset) \prod_{i=n+1}^{\infty} \frac{\alpha_{i}}{\alpha_{i}+\beta_{i}}
$$

for $n \geq k$, where $[$ see $(4.3)]$

$$
r_{i}(t, s, \emptyset)= \begin{cases}p_{i}(t), & \text { if } G_{i}(s)=0 \\ q_{i}(t), & \text { if } G_{i}(s)=1\end{cases}
$$

Since $\lim _{t \rightarrow 0} p_{i}(t)=\lim _{t \rightarrow 0} q_{i}(t)=1$ for all $i \geq 1$, we have $\liminf _{t \rightarrow 0} p_{\emptyset}(t, s) \geq$ $\prod_{i=n+1}^{\infty} \frac{\alpha_{i}}{\alpha_{i}+\beta_{i}}$, and the latter product may be chosen as close to 1 as desired. This shows that $\lim _{t \rightarrow 0} p_{\emptyset}(t, s)=1$. Therefore, since $\left\|\delta_{s^{\prime}}\right\|=1$ for all $s^{\prime} \in S$,

$$
\left\|T(t) \delta_{s}-\delta_{s}\right\| \leq\left\|\left(p_{\emptyset}(t, s)-1\right) \delta_{s}\right\|+\sum_{E \neq \emptyset} p_{E}(t, s)=2\left(1-p_{\emptyset}(t, s)\right) \quad(\text { use }(4.6))
$$

converges to 0 , as $t \rightarrow 0$.

Finally, assume

$$
\sum_{n=1}^{\infty} \beta_{n}=\infty
$$

[note that this assumption does not contradict (4.4)], and consider $s \in S$. Let $k$ be so large that all coordinates of $s$ with indexes $n \geq k$ are zero. For such $n, B_{n} \delta_{s}$ is given by the first formula in (4.1). Since all $F_{n}(s)$ 's are pairwise different and different from $s$, it follows that for $n \geq k$

$$
\left\|A_{n} \delta_{s}\right\|=\left\|\sum_{i=1}^{n} B_{i} x\right\| \geq \sum_{i=1}^{k-1} \gamma_{i}+\sum_{i=k}^{n} \beta_{i}
$$

where $\gamma_{i}$ is either $\beta_{i}$ or $\alpha_{i}$, depending on whether $G_{i}(s)=0$ or $G_{i}(s)=1$. In any case, $\lim _{n \rightarrow \infty}\left\|A_{n} \delta_{s}\right\|=\infty$. In other words, no $\delta_{s}$ belongs to the extended limit of the generators $A_{n}$. This explains why it is difficult to use the SovaKurtz theorem here: All members of $D\left(A_{\mathrm{e} x}\right)$ are 'skew' to the members of the natural basis. As a result, it is also quite hard to characterize the generator of the limit semigroup. 
To summarize: Blackwell's example is presented in the context of the infinite product of semigroups of Section. 2. In this example, it is hard to prove the existence of the limit semigroup using Theorem 2.1 but it is quite easy to prove it directly. All states $s$ of the Markov chain involved are instantaneous, which in terms of the semigroup means that none of the $\delta_{s}$ belongs to the domain of the generator. As a result, a manageable characterization of the generator of the semigroup is missing.

Open Access. This article is distributed under the terms of the Creative Commons Attribution License which permits any use, distribution, and reproduction in any medium, provided the original author(s) and the source are credited.

\section{References}

[1] W. Arendt et Al. Vector-valued Laplace Transforms and Cauchy Problems, Birkhäuser, Basel, 2001.

[2] W. Arendt, A. Driouich, and O. El-Mennaoui, On the infinite product of $C_{0}$-semigroups, J. Functional Analysis 160 (1998), 524-542.

[3] D. Blackwell, Another countable Markov process with only instantaneous states, Ann. Math. Statist 29 (1958), 313-316.

[4] A. Bobrowski, Functional Analysis for Probability and Stochastic Processes, Cambridge University Press, Cambridge, 2005.

[5] P. Cannarsa and G. Da Prato, On a functional analysis approach to parabolic equations in infinite dimensions, J. Functional Analysis 118 (1993), 22-42.

[6] R. L. Dobrushin, An example of a countable homogeneous Markov process all states of which are instantaneous, Teor. Veroyatnost. i Primenen. 1 (1956), 481-485.

[7] K.-J. Engel and R. Nagel, One-Parameter Semigroups for Linear Evolution Equations, Springer, New York, 2000.

[8] S. N. Ethier and T. G. Kurtz, Markov processes. Characterization and convergence, Wiley, New York, 1986.

[9] W. Feller and H. P. McKean, Jr., A diffusion equivalent to a countable Markov chain, Proc. Nat. Acad. Sci. U.S.A. 42 (1956), 351-354.

[10] D. Freedman, Markov Chains, Holden-Day, Inc., 1971.

[11] J. A. Goldstein, Semigroups of Linear Operators and Applications, Oxford University Press, New York, 1985.

[12] M. Hasegawa, A note on convergence of semi-groups of operators, Proc. Japan Acad. 40 (1964), 262-266.

[13] H. Heyer, Probability measures on locally compact groups, Springer-Verlag, Berlin-New York, 1977, Ergebnisse der Mathematik und ihrer Grenzgebiete, Band 94.

[14] G. A. Hunt, Semi-groups of measures on Lie groups, Trans. Amer. Math. Soc. 81 (1956), 264-293. 
[15] T. Kato, Perturbation theory for linear operators, Classics in Mathematics Series, Springer, 1995, reprint of the 1980 edition.

[16] T. G. KurTz, Extensions of Trotter's operator semigroup approximation theorems, J. Functional Analysis 3 (1969), 354-375.

[17] T. G. Kurtz, A general theorem on the convergence of operator semigroups, Trans. Amer. Math. Soc. 148 (1970), 23-32.

[18] T. M. Liggett, Continuous time Markov processes. An introduction, Amer. Math. Soc., 2010.

[19] A. C. McBride, Semigroups of linear operators: an introduction, Pitman Research Notes in Mathematics Series, vol. 156, Longman Scientific \& Technical, Harlow; John Wiley \& Sons, Inc., New York, 1987.

[20] R. NAGEL (ED.), One-parameter semigroups of positive operators, Lecture Notes in Mathematics, vol. 1184, Springer, 1986.

[21] M. Sova, Convergence d'opérations linéaires non bornées, Rev. Roumaine Math. Pures Appl. 12 (1967), 373-389.

[22] A. D. Wentzell, A course in the theory of stochastic processes, McGrawHill International Book Co., New York, 1981, Translated from the Russian by S. Chomet, With a foreword by K.L. Chung.

[23] D. Williams, Probability with martingales, Cambridge Mathematical Textbooks, Cambridge University Press, Cambridge, 1991.

[24] K. Yosida, Functional analysis, Springer, 1965.

\author{
AdAm Bobrowski \\ Lublin University of Technology, \\ Nadbystrzycka 38A, \\ 20-618 Lublin, \\ Poland \\ e-mail: a.bobrowski@pollub.pl
}

Received: 30 October 2014 